\title{
A Morphological Study of Ferritin Synthesis in Macrophages with Ingested Ferric Hydroxide-Potassium Polyvinyl Sulfate Complexes
}

\author{
Toshiro Ono ${ }^{1}$, Tadashi Tsujii ${ }^{2}$ and Satimaru Seno ${ }^{1}$ \\ ${ }^{1}$ Division of Pathology, and ${ }^{2}$ Division of Ultrastructure Research, Shigei \\ Medical Research Institute, 2117 Yamada, Okayama, 701-02, Japan
}

\begin{abstract}
A ferric hydroxide-polyvinyl sulfate colloidal solution (FePVS), prepared by mixing potassium polyvinyl sulfate (PVSK) and ferric hydroxide colloidal solution was used to study ferritin synthesis in rat peritoneal macrophages. The colloidal particles had spherical electron opaque ferric hydroxide cores with diameters of about $250 \mathrm{~nm}$ surrounded by radially arranged fibrous PVS molecules. They also had strong negative electric charges.

Fe-PVS particles injected into the peritoneal cavity were taken up by the macrophages then disintegrated rapidly. In the phagolysosomes the electron opaque ferric hydroxide cores of Fe-PVS were denuded of their PVS frames then decomposed into small 5-6 nm granules 24 to $48 \mathrm{~h}$ after injection. These small granules were released from the lysosomes into the hyaloplasm and the myelin figures were found in the lysosomal vacuoles. No reaccumulation of granules in lysosomes was found even 3 months later.

The intracellular distribution of ferritin in macrophages demonstrated by the immunocytochemical method showed a pattern similar to that of the small granules formed by the disintegration of Fe-PVS. This means that in rat peritoneal macrophages that contain ingested Fe-PVS particles ferritin first is synthesized in phagolysosomes by the ferric hydroxide cores that conjugate with apoferritin or protein subunits then they are dispersed into the cytoplasm. Two possible pathways for the biosynthesis of ferritin are discussed.
\end{abstract}

Ferritin is a ferric hydroxide-apoferritin complex that is widely distributed in animal bodies and serves as a storehouse for iron $(13,21)$. Heavy deposition of ferritin is induced easily in animals by injecting them with and excess of iron compounds $(12,19,20)$. Thus, the process of ferritin formation can be analyzed by using animal cells loaded with iron.

Recent studies of liver cells and hepatocytes given iron compounds in vivo as well as in vitro have greatly expanded our knowledge of ferritin synthesis.

It is generally believed that apoferritin is synthesized mainly in the cytoplasmic matrix by polysomes and that ferritin is formed there from the apoferritin by the incorporation of $\mathrm{Fe}(\mathrm{II})(6)$, after which the synthesized ferritin molecules accumulate in autophagosomes. Recently, it has been demonstrated that macrophages and

\footnotetext{
Abbreviations used: Fe-PVS, ferric hydroxide-potassium polyvinyl sulfate complex; PVSK, potassium polyvinyl sulfate; PBS, phosphate buffered saline; ER, endoplasmic reticulum.
} 
Kupffer cells in rat liver synthesize ferritin in the same way as do liver parenchymal cells (4).

Whether these two types of cells that have completely different functions have the same pathway for ferritin synthesis is not clear; macrophages have marked phagocytic activity and contain the basic proteins and enzymes that digest phagocytized materials. The iron compounds taken into the phagosomes of the macrophages inevitably are exposed to these active substances which hepatocytes lack. Macrophages take up gross particles of iron hydroxide colloidal particles, red cells and hemoglobin-haptoglobin complexes and use their iron as for ferritin synthesis.

Liver cells take up only specific iron compounds such as transferrin which is taken up by receptors on the cell surface (1). Therefore, there should be some difference in the pathway by which ingested iron compounds are synthesized into ferritin between macrophages and hepatocytes, because iron compounds can be taken up by macrophages but not by hepatocytes.

The ferritin molecule is visible by electron microscopy, and apoferritin can be detected by immunocytochemistry. Therefore, the synthetic pathway in macrophages can be analyzed morphologically if we use visible colloidal iron particles that are taken up specifically by macrophages and used the source of iron for ferritin synthesis.

Ferric hydroxide-polyvinyl sulfate colloidal particles (Fe-PVS) are an excellent tool for this analysis. They have a spherical and electron dense iron hydroxide core of about $250 \mathrm{~nm}$ that is surrounded by PVS molecules. They also give a strong positive Prussian blue reaction which makes them visible with electron microscopy as well as under light microscopy. In addition, these particles are taken up preferentially by macrophages and disintegrate rapidly into minute iron hydroxide particles in the phagolysosomes of rat peritoneal macrophages (16). These particles are taken up specifically by macrophages, but by no other somatic cells including hepatocytes.

We here show that rat peritoneal macrophages take up Fe-PVS particles and synthesize ferritin in the phagolysosomes very rapidly then discharge the ferritin into the cytoplasmic matrix with, or without, lysis of the limiting membrane. These ferritin particles that are dispersed diffusely into the hyaloplasm never reaccumulate in the autophagolysosomes.

\section{MATERIALS AND METHODS}

Preparation of Fe-PVS. The colloidal solution of Fe-PVS was prepared by adding 1 volume of ferric hydroxide colloidal solution, $0.8 \mathrm{mg} \mathrm{Fe} / \mathrm{ml}$ to 10 volumes of $2.5 \times 10^{-3} \mathrm{~N}$ potassium polyvinyl sulfate (PVSK) drop by drop with gentle stirring. The $\mathrm{pH}$ of the solution was adjusted to 7.3 by adding $1 \times 10^{-2} \mathrm{M}$ sodium bicarbonate, after which it was made isotonic by adding glucose then filtered through a $450 \mathrm{~nm}$ filter. The final concentration of iron was $0.044 \mathrm{mg} \mathrm{Fe} / \mathrm{ml}$. The colloidal iron solution was prepared just before use. The PVSK used for colloid titration $(15,24)$ was obtained from Wako Pure Chemical Indust. Ltd., Osaka.

Animals. Thirty adult male albino rats of the Wistar strain that weighed $250-300 \mathrm{~g}$ were used. They were divided into 8 groups, 3 animals each in groups 1 to 7 and 9 animals in group 8.

Intraperitoneal injection of Fe-PVS. Three milliliters of Fe-PVS solution was injected into the peritoneal cacity of each animal in groups 1 through 7 . The animals belonging to 
group 8 served as controls; each received one intraperitoneal injection of $3 \mathrm{ml}$ of $2.5 \times 10^{-3} \mathrm{~N}$ PVSK. Ascites cells were collected from animals in groups 1 through 6 at 1, 5, 24, 48, 120, and $144 \mathrm{~h}$, respectively after Fe-PVS injection. In animals belonging to group 7, ascites cells were taken 3 months after the Fe-PVS injection. Cells were obtained from 9 control animals, 3 animals at a time, 24, 72, and $144 \mathrm{~h}$ after PVSK injection. The ascites fluid obtained from the 3 animals in each group was combined and mixed then used in the experiments. Each experiment was repeated 3 times.

Preparation of antibody to ferritin. Rabbit antiserum to ferritin was prepared with an injection of rat liver ferritin emulsified with complete Freund's adjuvant. Rabbit antibody specific to ferritin was obtained and purified by affinity chromatography as follows: Ferritin was covalently linked to BrCN-activated Sepharose 4B (0.4 mg protein/ml Sepharose) (11). The antiserum to ferritin was added to the Sepharose $4 B$ column, which then was washed with PBS until the eluate showed no absorbance at $280 \mathrm{~nm}$.

The bound antibodies were eluted with $2 \mathrm{M}$ NaSCN. The effluent was collected and dialyzed exhaustively against PBS. The immunodiffusion test was done by the Ouchterlony double diffusion method. Rat liver ferritin was given by Dr. R. Matsuura (Okayama University Medical School).

Light microscopy of ascites smears. One droplet of the ascites fluid was smeared on a slide and used for light microscopy. The smear was fixed in methanol then treated with potassium ferrocyanide for the Prussian blue reaction, after which it was post-stained with hematoxylin.

Electron microscopy of peritoneal macrophages. Cell pellets obtained by centrifuging the ascites cells for $10 \mathrm{~min}$ at $900 \mathrm{rpm}$, were used for electron microscopy. The sedimented cells were fixed in $2 \%$ glutaraldehyde for $1 \mathrm{~h}$ at $4{ }^{\circ} \mathrm{C}$. After fixation these cells were suspended in $5 \times 10^{-2} \mathrm{M}$ sodium cacodylate buffer at $\mathrm{pH} 7.3$ then washed with the same solution. The cells then were post-fixed in $2 \% \mathrm{OsO}_{4}$ for $1 \mathrm{~h}$ after which they were dehydrated through graded ethanols to acetone then embedded in TAAB resin. Thick sections of about $2 \mu \mathrm{m}$ and subsequent thin sections of silver color were cut with a Reichert OmU-3 ultramicrotome. The thick sections were used for light microscopy and the thin ones for electron microscopy. The thin sections were observed in a Hitachi electron microscope, H-700, with or without staining by uranyl acetate and lead citrate.

Identification of ferritin by immunofluorescence. The indirect immunofluorescence method (14) was used. Smears of ascites cells fixed with methanol were rehydrated in PBS for $1 \mathrm{~min}$. These cells then were incubated for $30 \mathrm{~min}$ at room temperature with rabbit anti-rat ferritin antibody that had been purified by the method described above. After incubation the cells were washed with PBS and exposed to goat anti-rabbit fluorescein isothiocyanate-conjugated IgG (1:50 dilution in PBS, Cappel Laboratories Inc., Cochranville, Pa. U.S.A.) for $30 \mathrm{~min}$ then washed and observed under a fluorescence microscope. For the control, cells were incubated with normal rabbit serum in place of rabbit anti-ferritin antibody then stained with goat fluorescein-IgG.

\section{RESULTS}

Characteristics of Fe-PVS particles. Colloidal particles of Fe-PVS prepared by the method described had strong negative electric charges, $0.6 \mathrm{~mA} / \mathrm{cm}$ for $30 \mathrm{~min}$, when electrophoresed on cellulose acetate membranes $(22,23)$. Particles gave a positive Prussian blue reaction. Electron microscopy showed them as electron dense spherical particles about $250 \mathrm{~nm}$ in diameter that were framed by radially arranged fibrous PVS molecules (Fig. 1). 

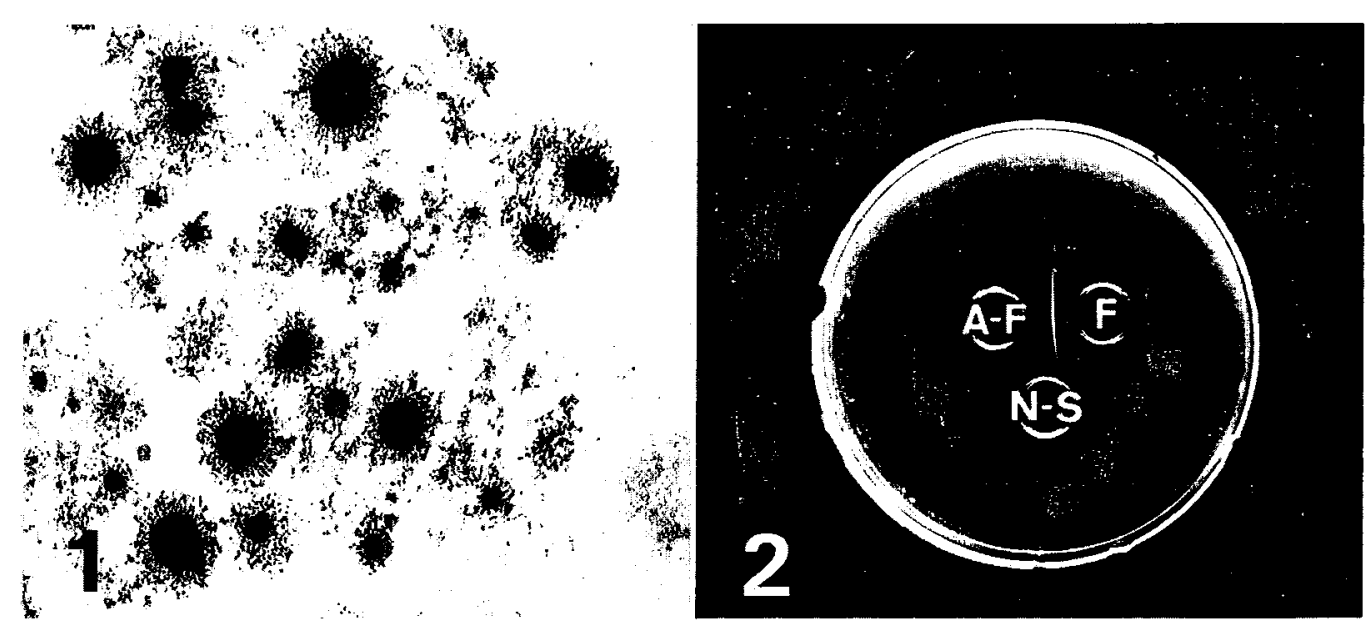

Fig. 1. EM photograph of Fe-PVS particles injected into the peritoneal cavity of a rat. Stain: uranyl acetate and lead citrate. $\times 50,000$.

Fig. 2. Ouchterlony immunodiffusion test made with rat ferritin and purified antibody. A-F: afinity purified anti-rat ferritin antibody. F: rat liver ferritin. NS: normal rabbit serum.
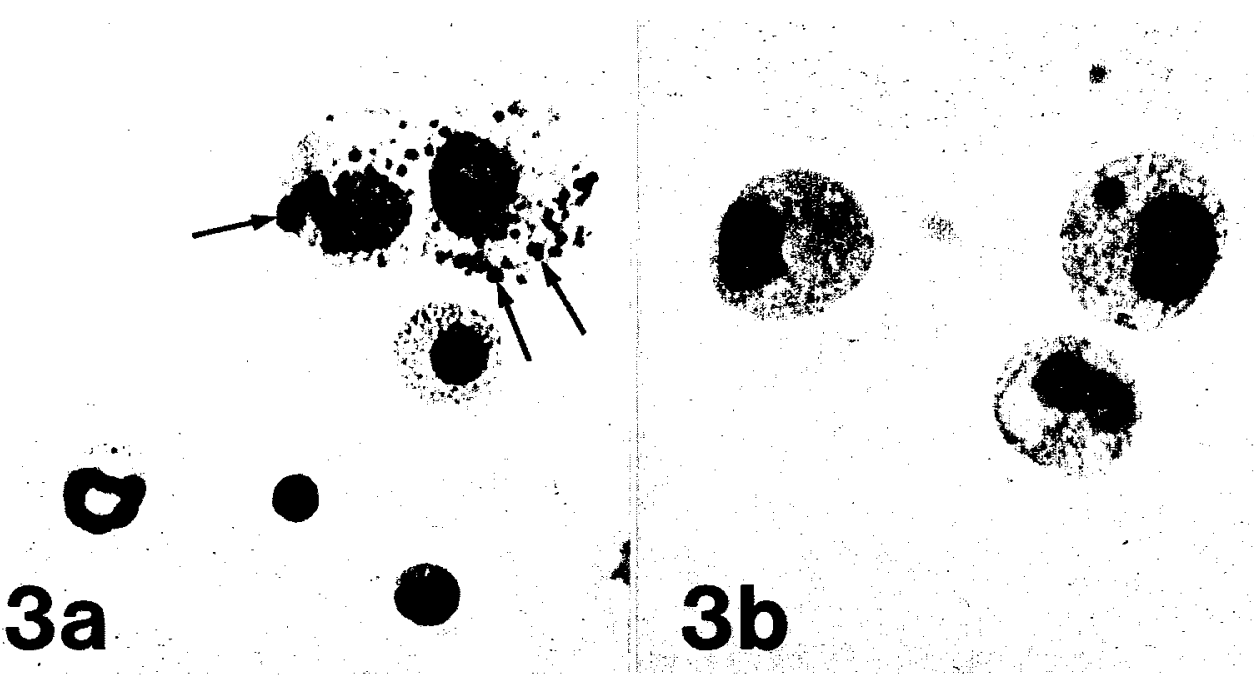

Fig. 3. LM photographs of rat ascites taken 24 (a) and $144 \mathrm{~h}$ (b) after the i.p. injection of FePVS. Cells were smeared and fixed then stained by the Prussian blue reaction and post-stained with hematoxylin. Fe-PVS showing a positive Prussian blue reaction appears as dark granules in the cytoplasm. (a) Peritoneal macrophages have Fe-PVS (arrows), but granulocytes, lymphocytes and mast cells do not. (b) Three peritoneal macrophages. Most granules in the cytoplasm had disappeared after 144 h. $\times 1,000$. 
Specificity of the antibody. The specificity of the purified antibody for ferritin was analyzed by immunodiffusion. A single precipitin line was formed between rat ferritin and the purified antibody (Fig. 2). No reaction took place between normal rabbit serum and the ferritin.

Light microscopy of ascites smears. Ascites cells collected $1 \mathrm{~h}$ after an i.p. injection of Fe-PVS had peritoneal macrophages that actively took up Fe-PVS as shown by the Prussian blue reaction. Most macrophages had several blue granules in their cytoplasms. Also, they had homogenous blue masses of Fe-PVS adhering to their cell surfaces. Macrophages taken $5 \mathrm{~h}$ after the Fe-PVS injection presented nearly the same picture, but the Fe-PVS masses on their cell surfaces were much reduced and the number of intracellular blue granules had increased. Twenty four hours after injection macrophages had lost the Fe-PVS on their surfaces and the intracellular blue granules tended to be large agglomerates (Fig. 3a). These agglomerates seemed to become smaller, but were more dense $48 \mathrm{~h}$ after the Fe-PVS injection. After $120 \mathrm{~h}$ the blue granules had grown much larger, but there were fewer of them. In addition, they had cytoplasmic vacuoles and gave a negative Prussian blue reaction. After $144 \mathrm{~h}$, the number of macrophages with cytoplasmic vacuoles had increased, most were filled with Prussian blue negative vacuoles and a small number of blue granules (Fig. 3b). After 3 months no macrophage that gave a positive Prussian blue reaction was found.

\section{Electron microscopy.}

1. Electron microscopy of sections stained with uranyl acetate and lead citrate. Observations of ascites cells on stained sections at specific intervals after Fe-PVS injection clearly showed the process of Fe-PVS uptake and decomposition by macrophages. Macrophages taken $1 \mathrm{~h}$ after the Fe-PVS injection had a number of Fe-PVS particles in their phagocytic vesicles and some particles adhering to their cell surfaces (Fig. 4a). Particles also were found in the phagosomes of macrophages sampled $5 \mathrm{~h}$ after Fe-PVS injection.

In some phagosomes the interparticular spaces appeared dark. These are the phagolysosomes formed by the fusion of phagocytic vacuoles with lysosomes (Fig. 4b). Twenty four hours after the Fe-PVS injection the number of phagolysosomes had increased. Each was smaller but its contents were denser. At this stage some myelin figures appeared in the phagolysosomes (Fig. 4c).

Forty eight hours after the Fe-PVS injection macrophages had large phagolysosomes containing Fe-PVS particles in various stages of disintegration and some myelin figures. In some phagolysosomes the limiting membrane became faint and its contents scanty, but there were some myelin figures and minute electron dense granules present (Fig. 4d). One hundred forty four hours after the Fe-PVS injection macrophages had a number of phagolysosomes, most with few contents, some had myelin figures with a few minute electron dense granules (Fig. 4e).

These results show that the Fe-PVS particles were attacked and decomposed by lysosomal enzymes in the phagolysosomes. Macrophages from controls given PVSK also had a large number of vacuoles. They also had myelin figures but no electron dense minute particles. This is evidence that Fe-PVS particles are decomposed in the phagolysosomes, the ferric hydroxide core being denuded of PVS, decomposed into minute granules then dispersed into the hyaloplasm.

2. Observations of unstained sections. Detailed information about the dis- 
integration of the ferric hydroxide core of Fe-PVS was obtained by observing unstained sections in which minute electron dense granules that were dispersed diffusely in cytoplasm could be seen clearly. Decomposition of the Fe-PVS particles took place in the phagolysosomes of the macrophages. Macrophages collected $5 \mathrm{~h}$ after the Fe-PVS injection had electron dense homogeneous ferric hydroxide cores of Fe-PVS in their phagolysosomes that were being disintegrated into minute granules 5-6 $\mathrm{nm}$ in diameter. Some of these granules also were found in the cytoplasmic matrix (Fig. 5a), but they were never present in the cytoplasm of macrophages from controls injected with PVSK and Hanks' solution.

A more advanced stage of the decomposition of the Fe-cores was seen in macrophages collected $48 \mathrm{~h}$ after the Fe-PVS injection. Small granules were well separated and were dispersed in the cytoplasmic matrix, except in the internal spaces of the mitochondria and endoplasmic reticulum (ER). The limiting membrane of the phagolysosomes often had disappeared (Fig. 5b). High magnification of the granules showed their individual shapes were like the iron-core of ferritin (Fig. 5b, inset).

At 120 and $144 \mathrm{~h}$ after the Fe-PVS injection, ferric hydroxide cores in advanced stages of decomposition were observed. The outline of the Fe-PVS core was very amorphous, and dense distribution of granules in the hyaloplasm was common. Numerous vacant vacuoles had been formed by the discharge of granules, but aggregates occasionally were present in the vacuoles (Fig. 6a).

Three months after the Fe-PVS injection, macrophages were similar to those collected $144 \mathrm{~h}$ after injection, but phagolysosomes were few and most of the small

Fig. 4. EM photographs of peritoneal macrophages taken 1 (a), 5 (b), 24 (c), 48 (d) and $144 \mathrm{~h}$ (e) after the i.p. injection of Fe-PVS. Cells were stained with uranyl acetate and lead citrate. A number of Fe-PVS particles with electron dense cores are present in the phogocytic vesicles ( $a, b, c$ and $d)$. (a) Fe-PVS particles in the phogosomes of the macrophages have nearly the same configuration as those found in intercellular spaces. (b) After fusion of the phagosomes with lysosomes (arrowhead) the PVS molecules surrounding the Fe cores became amorphous and denser (arrows). (c) Ferric hydroxide cores were denuded of their PVS coats when myelin figures formed (arrows). Note that neighboring lymphocytes have not ingested Fe-PVS particles (a and c). (d) Macrophage with decomposing Fe-PVS. The decapsulation of Fe-PVS particles at advanced stages is shown by the formation of distinct myelin figures in the phagolysosomes. (e) Macrophage with myelin figures (arrows) and some electron dense gross and minute particles in phago ysosomes. No Fe-PVS particles with original figures are present. There are few phagolysosomes $144 \mathrm{~h}$ after the Fe-PVS injection. a-c, $\times 11,000 ; d, \times 16,500$; e, $\times 15,000$.

Fig. 5. EM photographs of parts of the cytoplasms of peritoneal macrophages taken 5 (a) and $48 \mathrm{~h}$ (b) after the i.p. injection of Fe-PVS. These unstained sections clearly show the early stage of decomposition of the ferric hydroxide cores into small granules about 5-6 nm in diameter. Some granules are dispersed in the hyaloplasm (arrows), evidence that decomposition already had taken place $5 \mathrm{~h}$ after the Fe-PVS injection (a). Decomposition of ferric hydroxide cores proceeds sequentially (b). High magnification of minute particles in the hyaloplasm (inset in b). Unstained section. a and b, $\times 60,000$; inset in $b, \times 250,000$.

Fig. 6. EM photographs of parts of the cytoplasms of peritoneal macrophages taken $144 \mathrm{~h}$ (a) and 3 months (b) after the i.p. injection of Fe-PVS. The configuration of the lysosomal membrane has become faint (arrows) and ferritin particles are freely dispersed in the hyaloplasm. A few aggregated granules are present in some lysosomes (arrowhead) at $144 \mathrm{~h}$ of the experiment (a). Three months after the injection, ferritin particles are dense in only a few lysosomes, most granules have dispersed into the hyaloplasm (b). Particles in the lysosomes are residual. Most lysosomes are empty. No specific structures that would support the idea of the reaccumulation of granules in lysosomes are present. Unstained section. $\times 50,000$. 
Ferritin Synthesis in Peritoneal Macrophages

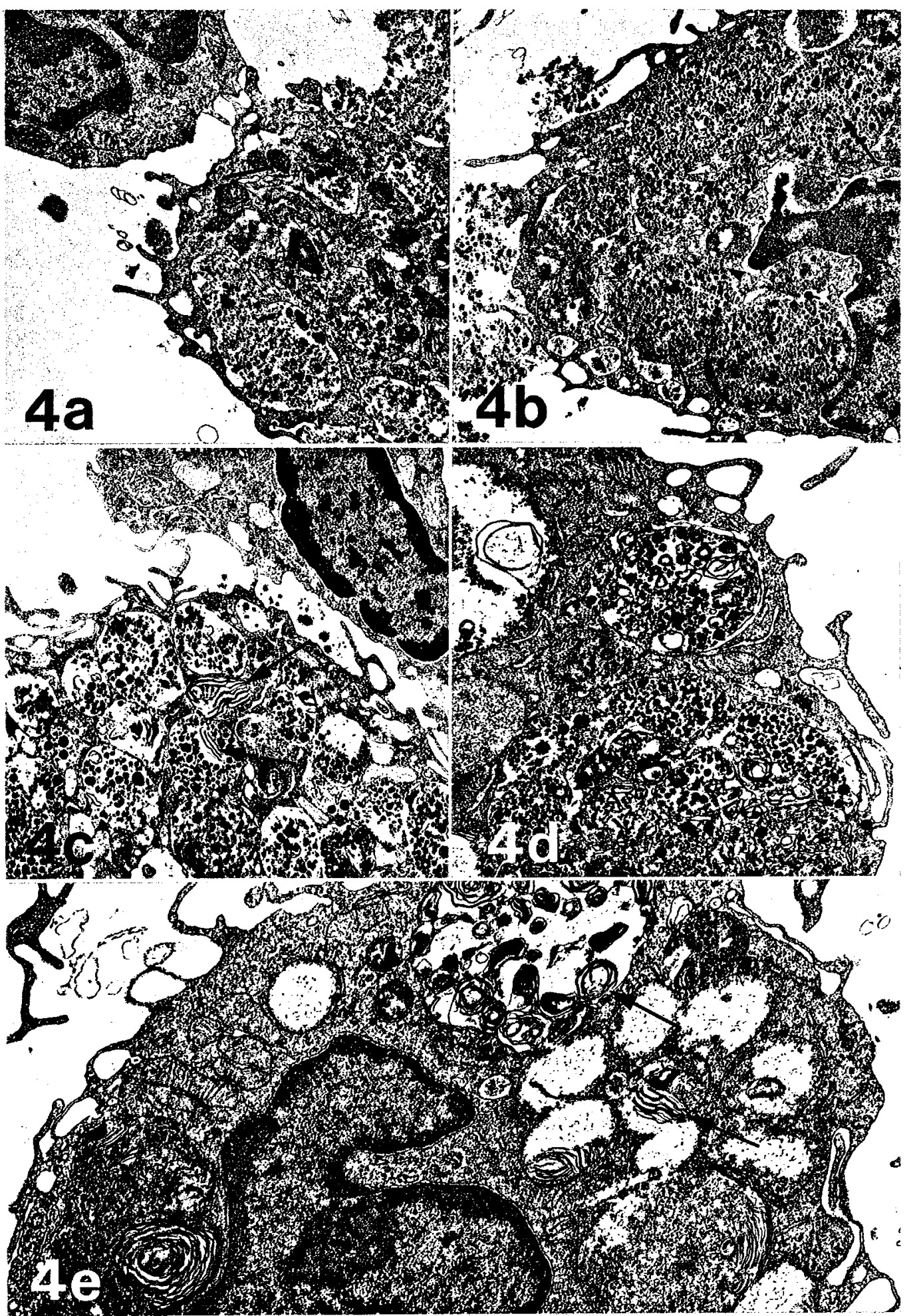




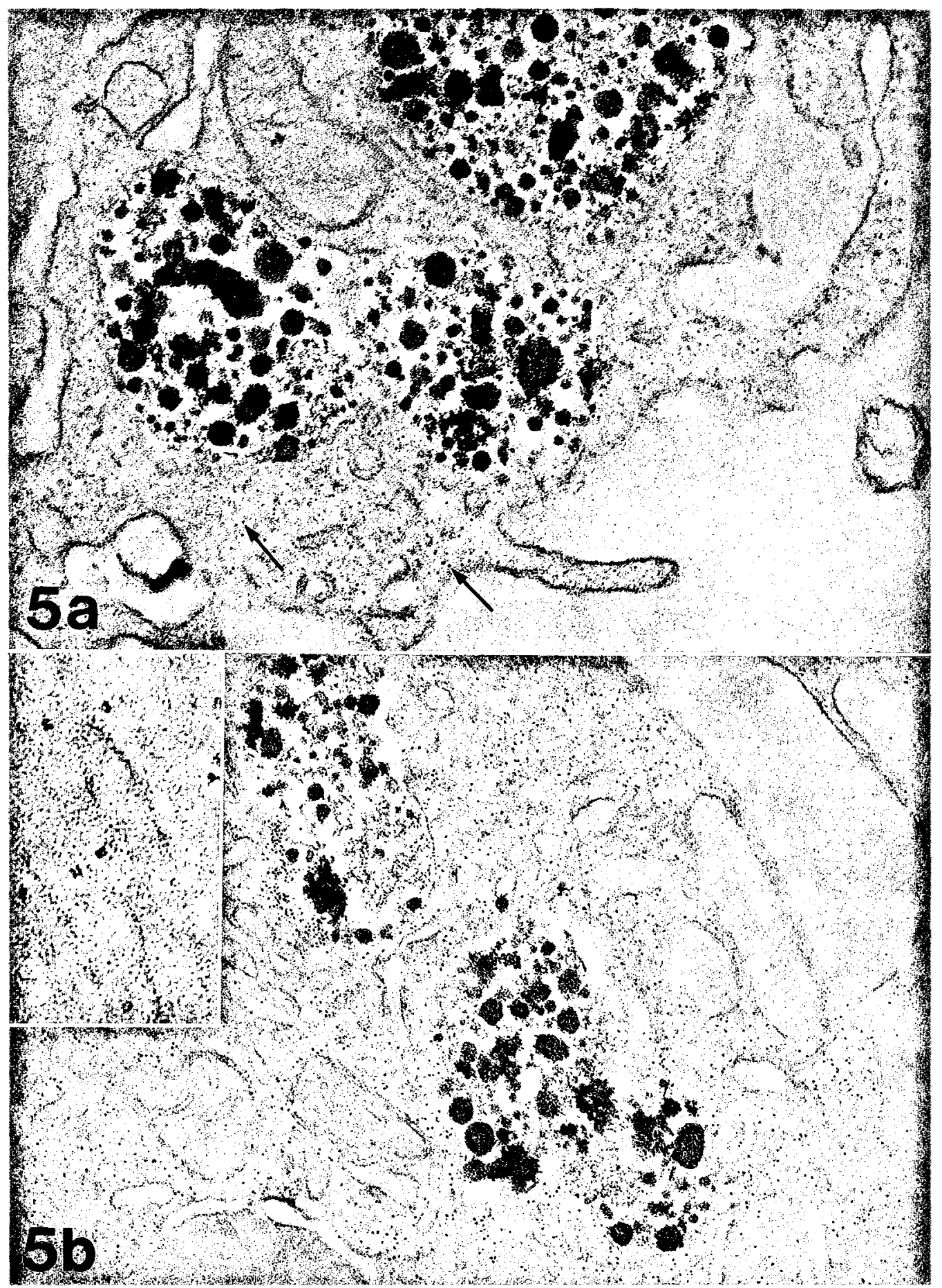




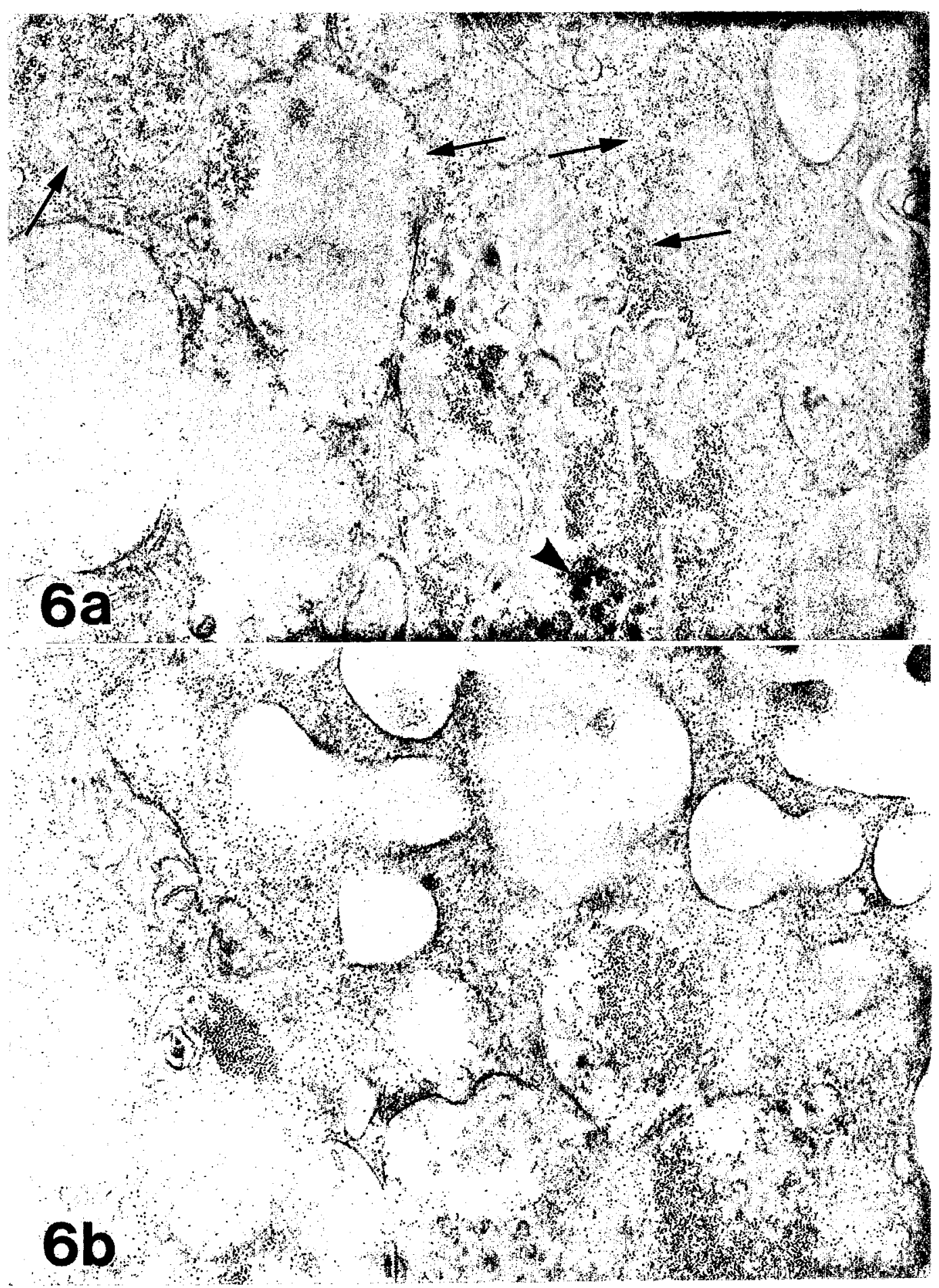



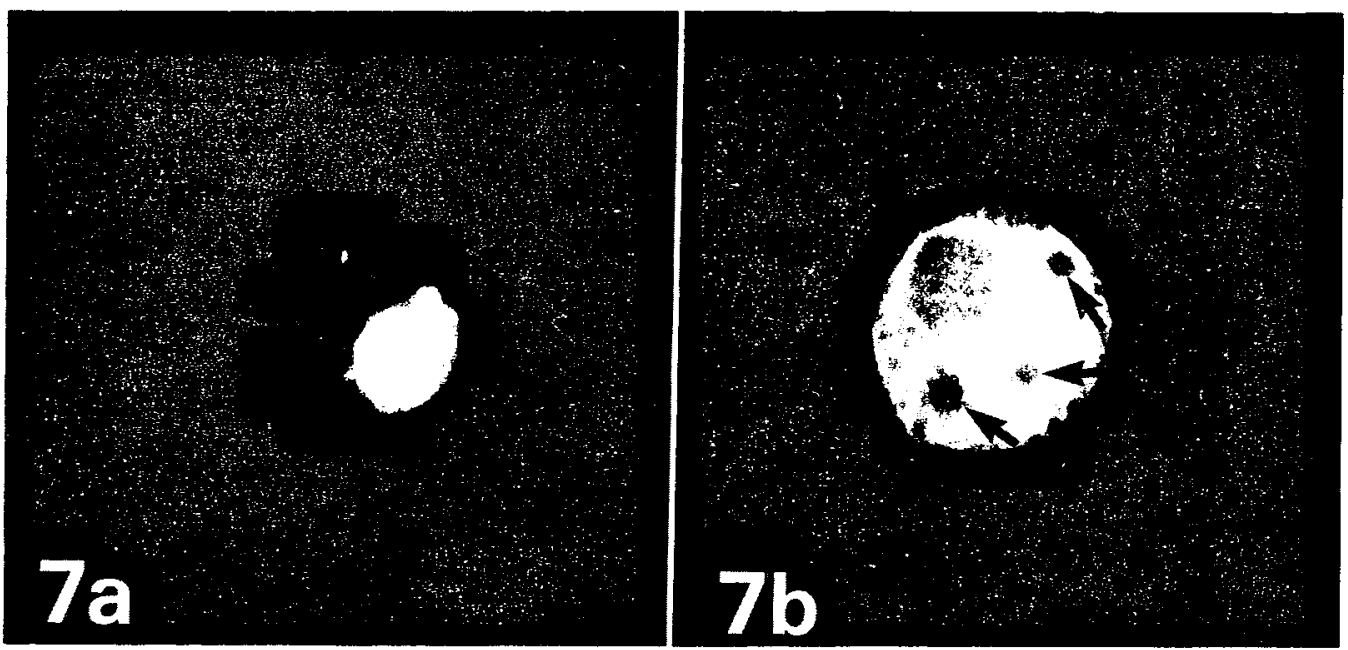

Fig. 7. Indirect immunofluorescence micrographs of ferritin distribution in macrophages taken 24 (a) and $120 \mathrm{~h}$ (b) after the i.p. injection of Fe-PVS. (a) Fluorescence is present in the cytoplasmic vacuoles in gross masses. (b) Fluorescence is diffuse in the cytoplasm, but is more intense than in a. No fluorescence is present in the cytoplasmic vacuoles (arrows). $\times 1,200$.

granules were found in the cytoplasmic matrix (Fig. 6b). Some vacuoles had granules dispersed diffusely but no microscopy showed any reaccumulation of granules in the autophagic vacuoles.

Immunofluorescence staining of ferritin in macrophages. Macrophages that had ingested Fe-PVS particles gave specific immunofluorescence for ferritin. In the period 24 to $48 \mathrm{~h}$ after the Fe-PVS injection macrophages stained with rabbit anti-rat ferritin antibody and goat anti-rabbit FITC IgG showed clear fluorescence on the vacuoles in their cytoplasms (Fig. 7a). The cytoplasmic matrix however, had hardly any fluorescence at this stage.

After 120 to $144 \mathrm{~h}$ macrophages had strong but diffuse fluorescence in the cytoplasm, indicating that ferritin was scattered diffusely throughout the cytoplasm (Fig. 7b). At this stage there was no vacuole fluorescence, evidence that ferritin had moved out of the vacuoles. Our results clearly show that ferritin first is synthesized in the phagolysosomes then is dispersed into the cytoplasmic matrix.

\section{DISCUSSION}

Our results clearly demonstrate that Fe-PVS particles taken into the phagolysosomes of the macrophages were decomposed very quickly. Disintegration started as early as $5 \mathrm{~h}$ after the Fe-PVS injection; it proceeded rapidly with lapse of time. Granules were dispersed into the cytoplasmic matrix with, or without, lysis of the limiting membrane of the phagolysosome. Finally, nearly all these granules were discharged from the phagolysosomes and were dispersed in dense patterns in the cytoplasmic matrix, where they remained for more than 3 months, no reaccumulation taking place in the cytoplasmic vacuoles. These newly formed and small electron dense granules are very similar in size and morphology to the iron core of ferritin. 
Immunofluorescence staining for ferritin showed that these granules actually are ferritin particles. This means that the Fe-PVS particles phagocitized by macrophages were decomposed rapidly to minute granules in the phagolysosomes then utilized for ferritin synthesis. Our findings are clear evidence that in macrophages ferritin first is formed in the phagolysosomes after which it is dispersed into the cytoplasmic matrix.

Our findings and conclusions do not agree with the current belief that apoferritin is synthesized mainly in the cytoplasmic matrix by polyribosomes $(7,18)$ and that ferritin first is formed in the cytoplasm by the incorporation of $\mathrm{Fe}(\mathrm{II})$ into the apoferritin molecules (6) then is translocated to the endolysosomes $(12,26)$.

This biosynthesis of ferritin has been demonstrated by loading lfepatocytes with an iron compound such as iron-dextran. Richter and his co-investigators stressed that approximately $85 \%$ of the apoferritin is synthesized by free polyribosomes and the remainder by membrane-attached polyribosomes $(9,10,17)$, and that finally ferritin molecules synthesized in the cytoplasmic matrix are accumulated in the endolysosomes through an autophagic mechanism $(5,8,25)$. The accumulation of ferritin molecules, which had been widely distributed in the cytoplasmic matrix, in endolysosomes must be an enormous, free energy-consuming reaction.

Although apoferritin molecules synthesized by membrane-attached polyribosomes reach the lysosomes by direct channeling through the ER and the Golgi apparatus $(3,25)$, Trump et al. showed that ferritin also is synthesized in hepatocytes by membrane-attached polyribosomes, then is released into the lumen of the rough ER and transported to lysosomes through the Golgi apparatus from the fact that they found ferritin molecules occasionally within the lumen of the rough ER and the Golgi cisternae (25). They also suggested that apoferritin molecules synthesized in the membrane-attached polyribosomes move to the Golgi apparatus then to the lysosomes where they incorporate iron to form ferritin $(2,25)$.

Our observations support the view that apoferritin, or its protein subunits, is synthesized by the membrane-attached polyribosomes and enters the lysosomes where it is synthesized to ferritin. We could not, however, obtain evidence that the 5-6 nm granules are decomposed into smaller ones and, finally, to invisible ferrous ion $[\mathrm{Fe}(\mathrm{II})]$ that then is incorporated into preexisting apoferritin molecules. These granules never disappeared, even briefly; they maintained the same size and morphology as the iron core of ferritin. The granules seemed to take up apoferritin molecules, or their subunits, and to organize ferritin molecules with no further decomposition.

Thus, the results of our experiments show that in peritoneal macrophages ferritin is synthesized with ferric hydroxide granules by taking up apoferritin molecules or their subunit proteins in the phagolysosomes, then the ferritin is dispersed diffusely into the cytoplasmic matrix. Whether peritoneal macrophages actually have a different pathway for ferritin synthesis from hepatocytes, or whether they use two different pathways for ferritin synthesis based on the chemical structure of the iron compounds taken into the cells is a matter for future study.

Acknowledgments. We thank Dr. H. Shigei, Chairman of the Board of the Shigei Medical Research Institute for his aid that made this study possible. We also are grateful to Dr. M. Awai, Okayama University Medical School, for his helpful suggestions and discussions and Dr. I. Urushizaki, Sapporo Medical College, for his valuable advice, and to Miss S. Ukita for her excellent technical assistance. 


\section{REFERENCES}

1. AISEN, P. The transferrins. in Iron in Biochemistry and Medicine, II, eds. A. Jacobs and M. Worwood, Academic Press, London, pp. 87-129, 1980

2. Arborgh, B.A.M., H. Glaumann and J.L.E. Ericsson. Studies on iron loading of rat liver lysosomes; effect on the liver and distribution and fate of iron. Lab. Invest. 30, 664-673, 1974

3. Arstila, A.U., W.D. Bradford, T.D. Kinney and B.F. TrumP. Iron metabolism and cell membranes. II. The relationship of ferritin to the cytocavitary network in rat hepatic parenchymal cell. Am. J. Pathol. 58, 419-449, 1970

4. Doolittle, R.L. and G.W. Richter. Isolation and culture of Kupffer cells and hepatocytes from single rat livers; with observations on iron-loaded Kupffer cells. Lab. Invest. 45, 558-566, 1981

5. Fedorko, M.E., N.L. Cross and J.G. Hirsh. Appearance and distribution of ferritin in mouse peritoneal macrophages in vitro after uptake of heterologous erythrocytes. J. Cell Biol. 57, 289-305, 1973

6. Harrison, P.M., G.A. Clegg and K. May. Ferritin structure and function. in Iron in Biochemistry and Medicine, II, eds. A. Jacobs and M. Worwood, Academic Press, London, pp. 131-171, 1980

7. Hicks, S.J. and H.N. Munro. Preferential synthesis of ferritin and albumin by different populations of liver polysomes. Science (Wash. D.C.) 164, 584-585, 1969

8. Hultcrantz, R., B.A.M. Arborgh, R. Wroblemski and J.L.E. Ericsson. Studies on the rat liver following iron overload. Electron probe X-ray microanalysis of acid phosphatase and iron. Am. J. Pathol. 96, 625-640, 1979

9. LEE, S.S.C. and G.W. RICHTER. Biosynthesis of ferritin in rat hepatoma cells and rat livers. I. Synthesis and assembly of protein subunits of ferritin. J. Biol. Chem. 252, 2046-2053, 1977

10. LEE, S.S.C. and G.W. RICHTER. Biosynthesis of ferritin in rat hepatoma cells and rat livers. II. Binding of iron by ferritin protein. J. Biol. Chem. 252, 2054-2059, 1977

11. March, S.C., I. Parikh and P. Cuatrecasas. A simplified method for cyanogen bromide activation of agarose for affinity chromatography. Anal. Biochem. 60, 149-152, 1974

12. Muir, A.R. and L. Golberg. Observations on subcutaneous macrophages. Phagocytosis of irondextran and ferritin synthesis. Q.J. Exp. Physiol. 46, 289-298, 1961

13. Munro, H.N. and M.C. Linder. Ferritin; structure, biosynthesis and role in iron metabolism. Physiol. Rev. 58, 317-396, 1978

14. NishiYa, K., J.M. Chiao and M. De Sousa. Iron binding proteins in selected human peripheral blood cell sets: immunofluorescence. Br. J. Haematol. 46, 235-245, 1980

15. Ono, T., H. Miyata and K. Toei. Polysoap as a new titrant for the determination of sodium dodecylbenzensulfonate by colloid titration. Bull. Chem. Soc. Jpn. 52, 425-427, 1979

16. Ono, T., S. UkitA, T. Tsuji and S. SEno. The clearance mechanism against foreign substances by living organisms. A study by using iron colloid particles of positive (ferric cacodylate, FeCac) and negative (ferric polyvinylsulfate, Fe-PVS) electric charges. Cell Struct. Funct. 7, 442, 1982

17. PURo, D.G. and G.W. Richter. Ferritin synthesis by free and membrane-bound polyribosomes of rat liver. Proc. Soc. Exp. Biol. Med. 138, 399-403, 1971

18. ReDman, C.W. Biosynthesis of serum proteins and ferritin by free and attached ribosomes of rat liver. J. Biol. Chem. 244, 4308-4315, 1969

19. RiCHTER, G.W. A study of hemosiderins with the aid of electron microscopy; with observations on the relationship between hemosiderin and ferritin. J. Exp. Med. 106, 203-218, 1957

20. Richter, G.W. The cellular transformation of injected colloidal iron complexes into ferritin and hemosiderin in experimental animals. J. Exp. Med. 109, 197-216, 1959

21. Richter, G.W. The iron-loaded cell; The cytopathology of iron storage. Am. J. Pathol. 91, 363-405, 1978

22. Seno, S., A. Tanaka, M. Urata, K. Hirata, H. Nakatsuka and S. Yamamoto. Phagocytic response of rat liver capillary endothelial cells and Kupffer cells to positive and negative 
charged iron colloid particles. Cell Struct. Funct. 1, 119-127, 1975

23. Seno, S., T. ONo and T. Tsujir. Macromolecular charge and cellular surface charge in adhesion, ingestion and blood vessel leakage. Ann. N.Y. Acad. Sci. 1983, in press.

24. Toei, K. and T. Kohara. A conductometric method for colloid titrations. Anal. Chim. Acta 83, 59-65, 1976

25. Trump, B.F., J.M. Valigorsky, A.U. Arstila, W.J. Mergner and T.D. Kinney. The relationship of intracellular pathways of iron metabolism to cellulan iron overload and the iron storage disease. Am. J. Pathol. 72, 295-336, 1973

26. ZeLIGS, J.D. Ultrastructure of the degradation of erythrocytes by thyroid epithelial cells in vivo. Am. J. Pathol. 89, 85-104, 1977 